\title{
Estimation of Internal Condition in Piled Fragment Rocks by Use of Resistive Forces Acting on the Bucket of Wheel Loaders
}

\author{
Hiroshi TAKAHASHI* \\ *Dept. of Geoscience and Technology, Graduate School of Engineering, \\ Tohoku University, Sendai 980-77, JAPAN \\ htaka@ecml.earth.tohoku.ac.jp
}

\begin{abstract}
In order to automate the scooping task, it is necessary to obtain the resistive forces acting on the bucket before the scooping is performed. In this study, empirical equations to predict the friction coefficient between the bucket and rocks, the friction coefficient among rocks and particle diameters were obtained by using the resistive forces which are measured at the beginning of the scooping task. In result, it became enable to predict the existence of huge rock inside of the rock pile by learning the resistive force which can be measured in the beginning of the scooping task.
\end{abstract}

\section{INTRODUCTION}

Recently, robotics has been receiving considerable attention in many fields, especially in mines and tunnel construction field[1]. Generally, the development process of the tunnel or levels in mines consists of drilling, blasting, scooping of the fragment rocks and removing of the rocks from the cutting face. The automation of above each process has already been tried, but the scooping is much more difficult to automate compared to other tasks because the shape of the rock pile changes according to the advance of the work. Furthermore, the interaction between the scooping tool(bucket) and crushed rocks is very complicated and it needs many parameters to describe.

In order to automate the scooping task, it is necessary to obtain the resistive forces acting on the bucket before the scooping is performed[2]. Therefore, some models to predict the resistive forces acting on bucket were proposed already[3,4]. But, as mentioned before, many parameters such as the friction coefficient between the bucket and rocks, the friction coefficient among rocks, slope of the rock pile and rock density are necessary to calculate the resistive force from these models. However, these parameters are generally unknown before the task. Therefore, the machine needs to obtain these parameters autonomously.

The purpose of this study is to propose a method to obtain the friction coefficient between the bucket and rocks, the friction angle among rocks and particle diameters by using the resistive forces which are measured at the beginning of the scooping task.

\section{EXPERIMENTAL APPARATUS AND PRODECURE}

Figure 1 shows the schematic diagram of an experimental apparatus. Two kinds of apparatus were used. One is a small size apparatus and the other is a medium size one. The sizes of the bucket in these apparatuses are shown in figure 1. The capacity of the 


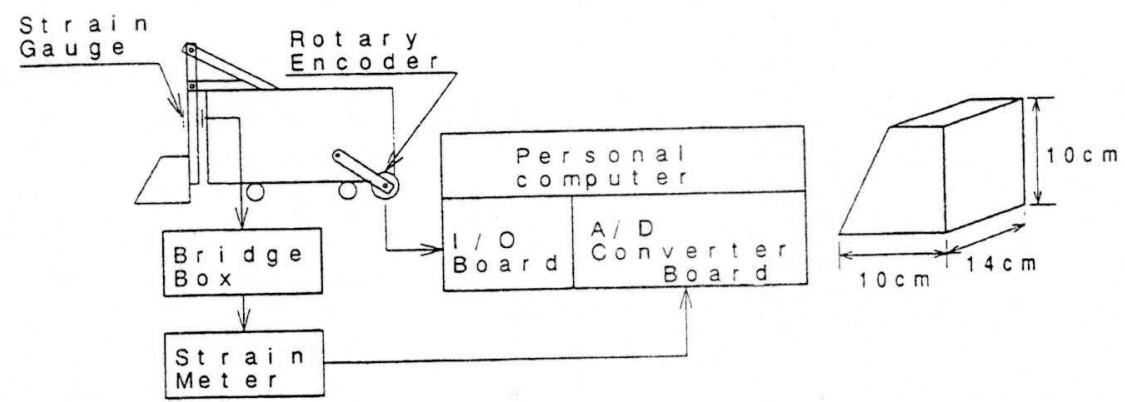

small size apparatus
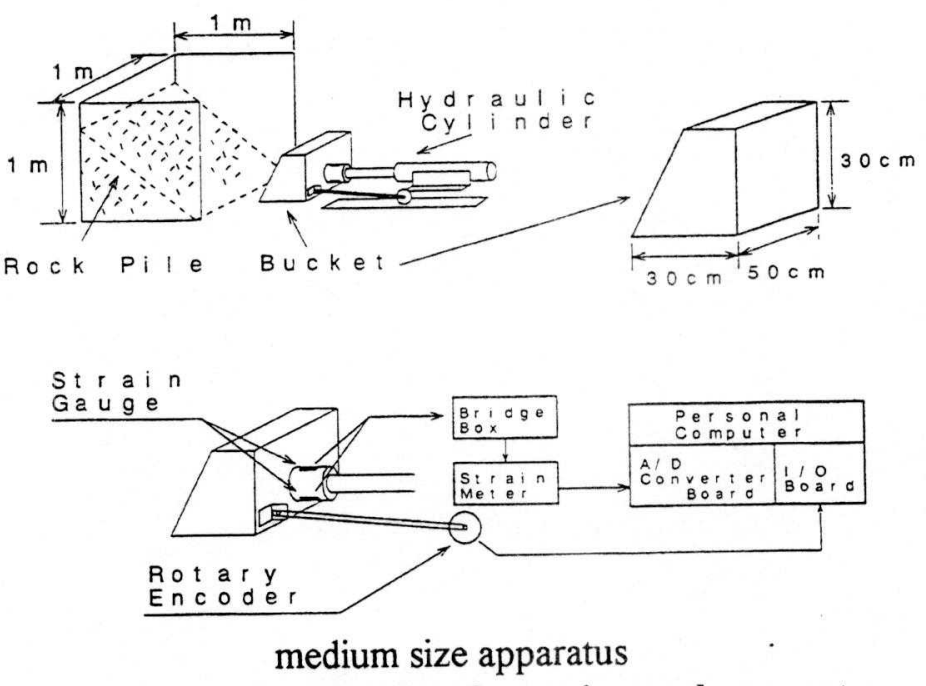

Fig.1 Schematic diagram of experimental apparatus

bucket in the medium size apparatus is about 32 times of the one in the small size apparatus.

The resistive force is a function of the penetration distance of the bucket into the rock pile. So, it is necessary to measure the penetration distance and resistive force simultaneously. To measure the resistive force, two strain gauges were attached on the lift arm in the small size apparatus. For the medium size apparatus, two strain gauges were attached on the surface of the acrylic cylinder and this cylinder was installed between the hydraulic cylinder and the bucket. The signals from the strain gauges were transmitted to the personal computer through the strain meter and A/D converter. The penetration distance was measured by the rotary encoder. The rotary encoder generates 100 pulses per one rotation. The distance was measured at every 5 pluses. As the diameter of the pinion is $28 \mathrm{~mm}$, the distance at one pulse is about $0.9 \mathrm{~mm}$.

The rocks used in this experiment were 13 kinds of crushed rock and one kind of quartz sand. Properties of particles are listed in Table 1. By using these particles, the rock pile was made with changing its slope.

\section{EXPERIMENTAL RESULTS}

Figure 2 shows the relationship between the resistive force, $\mathrm{F}$ and penetration 
Table 1 Properties of particles used in this experiment

\begin{tabular}{|c|c|c|c|c|c|c|}
\hline Rock & $\begin{array}{c}\text { Particle } \\
\text { diameter } \\
d_{s}[\mathrm{~mm}]\end{array}$ & $\begin{array}{c}\text { Rock } \\
\text { Density } \\
\rho\left[\mathrm{kg} / \mathrm{m}^{3}\right] \\
\end{array}$ & $\begin{array}{c}\text { Friction } \\
\text { Angle } \\
\phi_{k}[\mathrm{deg}]\end{array}$ & $\begin{array}{c}\text { Friction } \\
\text { Coefficient } \\
\mu[-]\end{array}$ & $\begin{array}{l}\text { Porosity } \\
\qquad \varepsilon[-]\end{array}$ & $\begin{array}{c}\text { Slope of } \\
\text { Rock Pile } \\
\phi[\text { deg] }\end{array}$ \\
\hline \multirow{13}{*}{ Crushed Rock } & 5.7 & \multirow{5}{*}{2740} & 35 & 0.56 & 0.47 & \multirow{14}{*}{34} \\
\hline & 2. 9 & & 34 & 0.54 & 0.49 & \\
\hline & 1.9 & & 33 & 0.53 & 0.52 & \\
\hline & 0.9 & & 32 & 0.514 & 0.53 & \\
\hline & $5.7+0.9$ & & 33.3 & 0.539 & 0.45 & \\
\hline & 20 & \multirow{5}{*}{2500} & 43 & 0.587 & 0.49 & \\
\hline & 12 & & 40 & 0.545 & 0.50 & \\
\hline & 8 & & 39 & 0.521 & 0.51 & \\
\hline & 5.8 & & 36 & 0.506 & 0.519 & \\
\hline & $13 \sim 4$ & & 40 & 0.533 & 0.48 & \\
\hline & 10 & 2610 & 40 & 0.537 & 0.48 & \\
\hline & $15 \sim 3$ & 2610 & 38 & 0.531 & 0.44 & \\
\hline & $12+5.8+0.9$ & 2570 & 38 & 0.542 & 0.423 & \\
\hline Quartz Sand & 0.7 & 2530 & 28 & 0.48 & 0.38 & \\
\hline
\end{tabular}

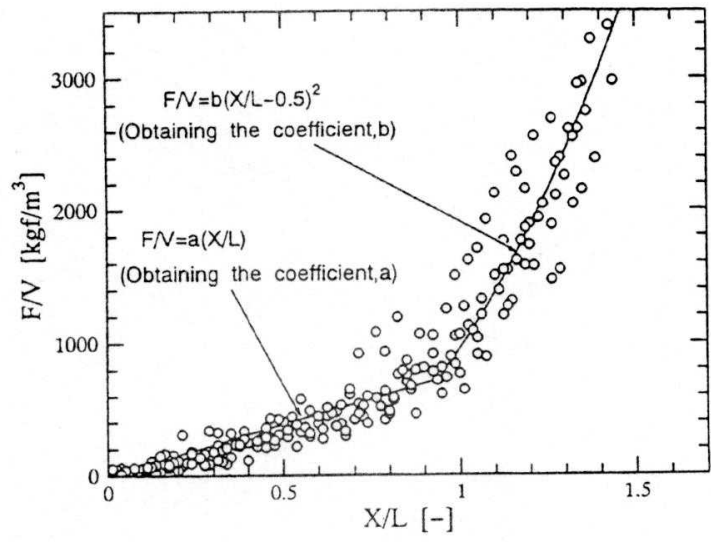

Fig. 2 Relationship between $X / L$ and F/V
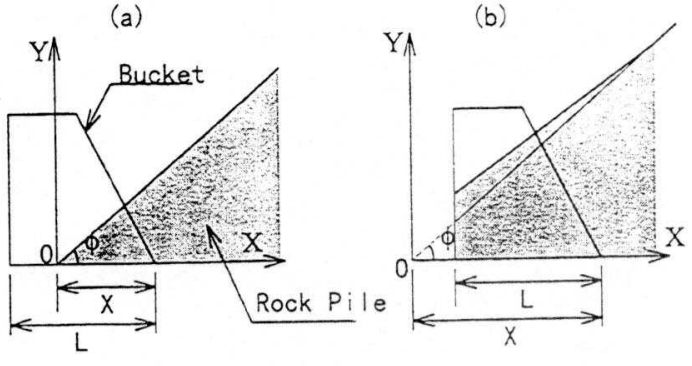

Fig.3 Penetration process of bucket into the rock pile

distance of the bucket into the rock pile, $\mathrm{X}$. The vertical axis shows the resistive force per unit bucket volume, F/V. The penetration distance was normalized by the bucket bottom length, $\mathrm{L}$. As shown in this figure, $\mathrm{F} / \mathrm{V}$ increased gradually with an increase of $\mathrm{X} / \mathrm{L}$ within the range of $\mathrm{X} / \mathrm{L}<1$. On the other hand, $\mathrm{F} / \mathrm{V}$ increased rapidly with increasing $\mathrm{X} / \mathrm{L}$ when $\mathrm{X} / \mathrm{L}$ exceeds unity. This trend was the same regardless of the rocks. Therefore, the relationship between the resistive force, $F / L$ was assumed to be a linear function in the range of $X / L<1$. On the other hand, in the range of $X / L>1$, the relationship between $\mathrm{F} / \mathrm{L}$ and $\mathrm{X} / \mathrm{L}$ was assumed to be a second order function. Therefore, the following equations were assumed.

$$
\begin{array}{ll}
\text { For } \mathrm{X} / \mathrm{L}<1 & F / V=a(X / L) \\
\text { For } \mathrm{X} / \mathrm{L}>1 & F / V=b(X / L-0.5)^{2}
\end{array}
$$

From the measured data, the coefficients, $a$ and $b$ were obtained.

Figure 3 shows the schematic diagram of penetration process of the bucket into the 
rock pile. For $\mathrm{X} / \mathrm{L}<1$, since the bottom and side of the bucket penetrates into the rock pile, it can be considered that the effect of the friction coefficient between the bottom and side of the bucket and rocks on the resistive force is significant. On the other hand, for $\mathrm{X} / \mathrm{L}>1$, the friction among rocks in the rock pile is significant. Therefore, in this study, the friction coefficient between the bucket and rocks is obtained by using the coefficient, a. Furthermore, the friction angle among rocks is obtained by using the coefficient, $b$. The particle diameter is obtained from both of them.

Figure 4 shows the relationship between the coefficient, a and the friction coefficient between the bucket and rocks, $\mu$. Figure 5 shows the relationship between the coefficient, $a$ and particle diameters, ds. It is found from figure 4 that the friction coefficient depends on the coefficient, a, slope of the rock pile, $\phi$ and particle density, $\rho$. Therefore, the friction coefficient is expressed by Eq.(3). Though the effect of particle density on particle diameter is not significant, Eq.(4) was assumed with considering Eq.(3).

$$
\begin{gathered}
\mu=f_{1}(a, \rho, \phi) \\
d s_{1}=f_{2}(a, \rho, \phi)
\end{gathered}
$$

From above equations, the following equations were assumed.

$$
\begin{aligned}
& \mu=K_{1} a^{\alpha 1} \rho^{\beta 1} \phi^{\gamma 1} \\
& d s_{1}=K_{2} a^{\alpha 2} \rho^{\beta 2} \phi^{\gamma 2}
\end{aligned}
$$

By using the measured resistive forces, following empirical equations to predict the friction coefficient and particle diameters were obtained in this study.

$$
\begin{aligned}
& \mu=0.13 a^{0.16} S^{1.25} \phi^{-0.25} \\
& d s_{1}=0.32 a^{1.76} S^{0.11} \phi^{-2.7}
\end{aligned}
$$

Here, $\mathrm{S}$ is the specific gravity of rocks. $\mathrm{S}$ is used in these equations instead of $\rho$ with considering the dimension of each hand side in Eq.(7).

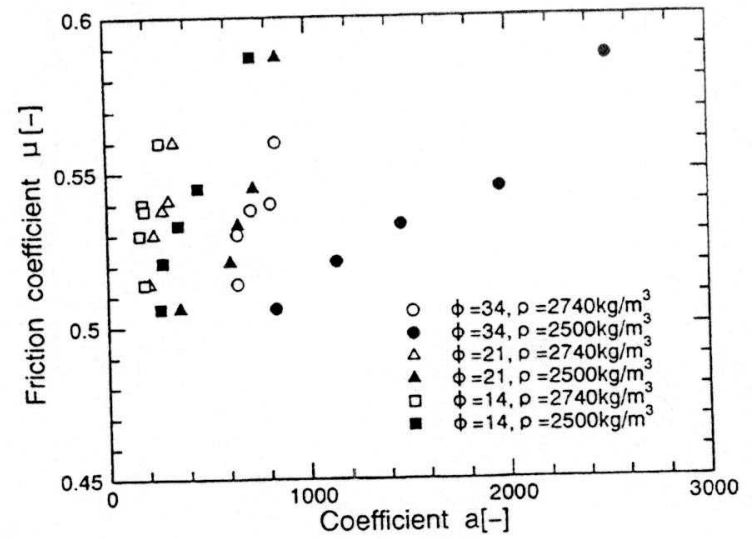

Fig. 4 Relationship between coefficient, a and friction coefficient, $\mu$

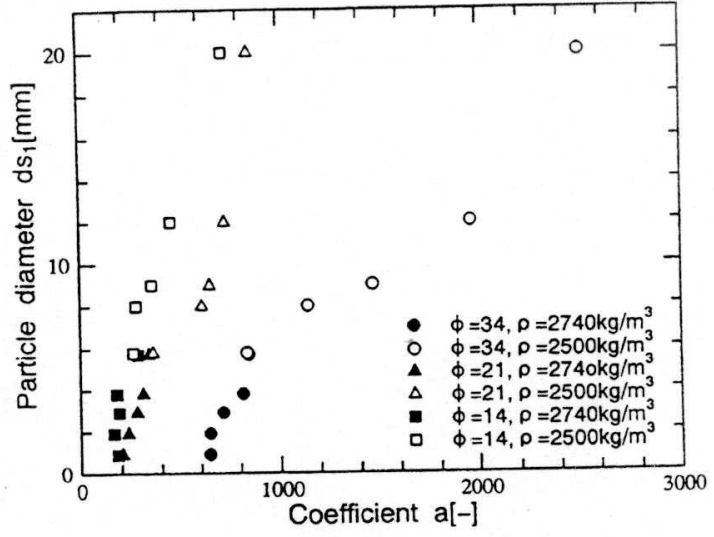

Fig.5 Relationship between coefficient, a and particle diameter, ds

Figure 6 shows the comparison between measured values and predicted ones for the friction coefficient and particle diameters. It shows a good agreement. 

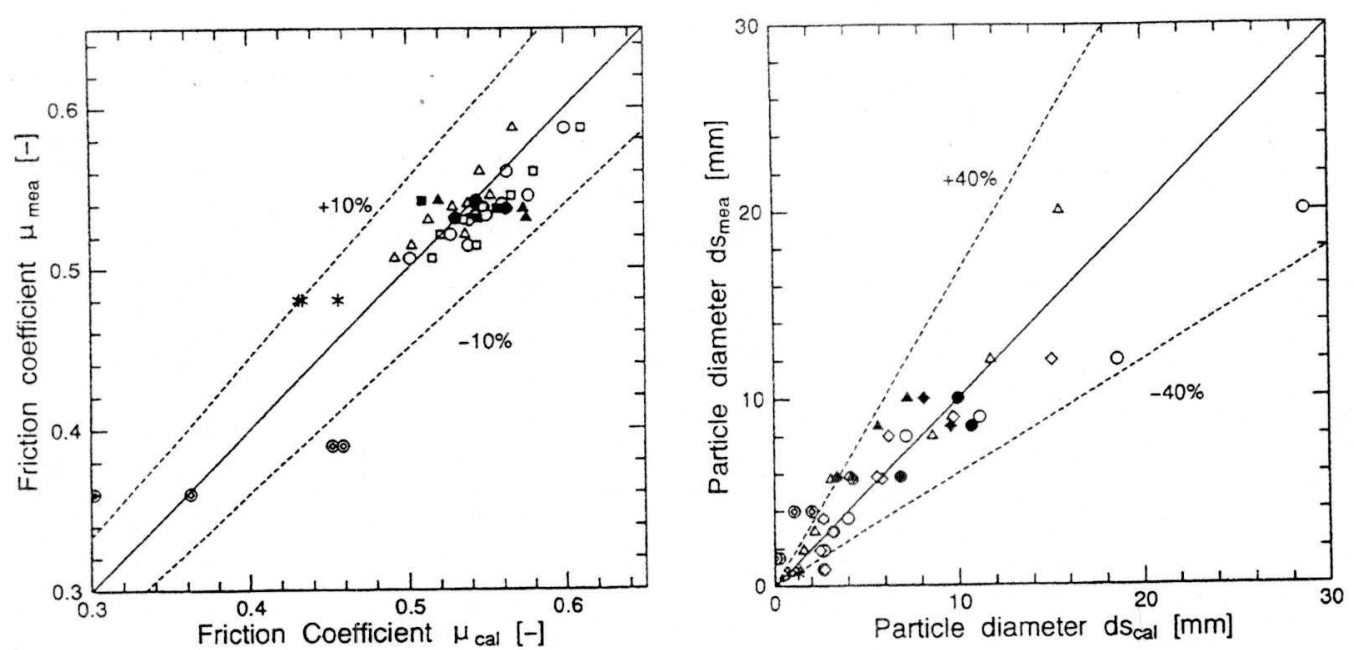

Fig.6 Comparison between measured values and predicted ones

Figure 7 shows the relationship between the coefficient, $\mathrm{b}$ and the friction angle among rocks, $\phi_{\mathrm{k}}$. The friction coefficient among rocks is given by $\tan \phi_{\mathrm{k}}$. In the field of soil mechanics, this angle is called internal friction angle of soil, and is often used in the soil analysis. In this study, the friction angle was used with taking the internal friction angle of soil into account. Figure 8 shows the relationship between the coefficient, $b$ and particle diameters. It is found from figure 7 that the effect of coefficient, $b$ and the slope of the rock pile on the friction angle among rocks is large, but the effect of particle density is not significant. Therefore, the friction angle among rocks is expressed by Eq.(9). For the particle diameters, Eq.(10) was assumed to match the form of Eq.(4).
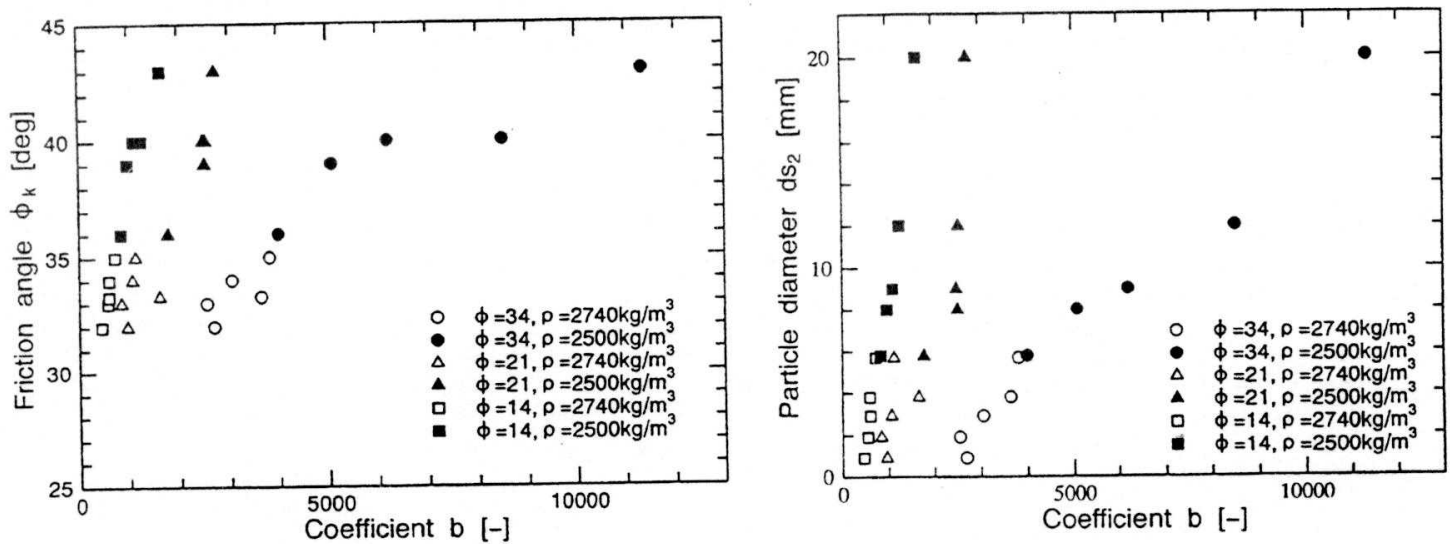

Fig.7 Relationship between coefficient, b Fig.8 Relationship between coefficient, b and friction angle among rocks, $\phi_{k}$ and particle diameter, $\mathrm{ds}$

$$
\begin{aligned}
& \phi_{k}=f_{3}(b, \phi) \\
& d s_{2}=f_{4}(b, \rho, \phi)
\end{aligned}
$$

From above equations, the following equations were assumed.

$$
\begin{aligned}
& \phi_{k}=K_{3}\left(b \cdot \phi_{S}\right)^{\alpha 3} \phi^{\gamma 3} \\
& d s_{2}=K_{4} b^{\alpha 4} S^{\beta 4} \phi^{\gamma 4}
\end{aligned}
$$


By using the measured resistive forces, following empirical equations to predict the friction angle among rocks and particle diameters were obtained

$$
\begin{aligned}
& \phi_{k}=20\left(b \cdot \phi_{S}\right)^{0.27} \phi^{-0.54} \\
& d s_{2}=1.24 b^{0.9} S^{-0.14} \phi^{-1.3}
\end{aligned}
$$

$\phi_{\mathrm{s}}$ in Eq.(11) shows the shape factor of rocks. The reason why $\phi_{\mathrm{s}}$ is included in Eq.(11) is that the friction among rocks depends on the roughness of the rock shape. In this study, the shape factor, $\phi_{\mathrm{s}}$ is defined by the ratio of maximum length to the minimum length of the rock. The shape factor of the crushed rock used in this study was about 2.5 .

Figure 9 shows the comparison between measured values and predicted ones for the friction angle among rocks and particle diameters. Again, it shows a good agreement.
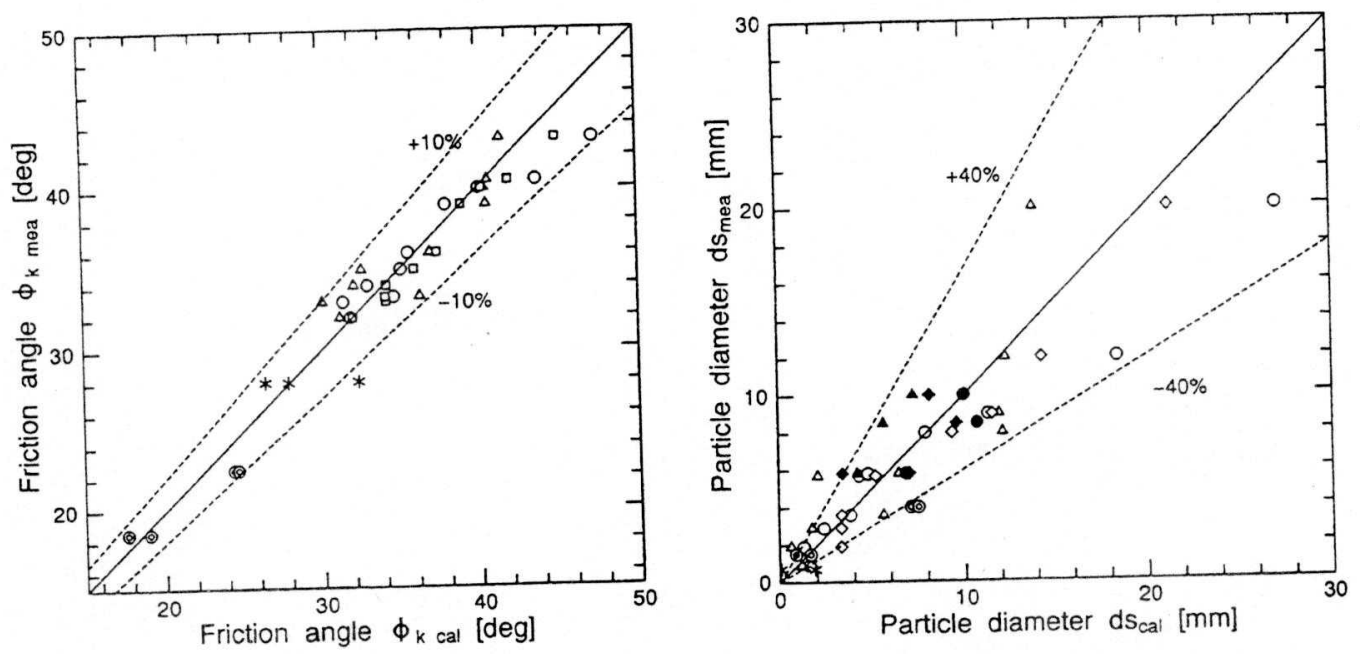

Fig.9 Comparisons between measured values and predicted ones

The measurement of resistive forces when the huge rock is included in the rock pile was carried out. In order to investigate the effect of the huge rock location on the resistive force, the following three patterns were examined as shown in figure 10 .

(a) The huge rock is on the bottom of the rock pile.

(b) The huge rock is in the middle of the rock pile.

(c) The huge rock is in the side of the rock pile.

Figure 11 shows the comparison between the resistive force with and without containing the huge rock. Black and white symbols indicate the resistive forces with and without containing the huge rock, respectively. When the huge rock is on the bottom of the rock pile, the resistive force is large compared to the one without huge rock, because the bucket tip hits the huge rock and pushes it. When the bucket tip hits and pushes the huge rock, the cutting force becomes large. Consequently, the resistive force becomes large. When is the huge rock is in the middle of the rock pile, the resistive force is large. But the resistive force is less than that in case (a), because the bucket tip does not push the huge rock. In the case of (c), the resistive force becomes large when the bucket tip hits the huge rock. However, as the huge rock is not included inside of the bucket, the resistive force approaches to the one without the huge rock. 

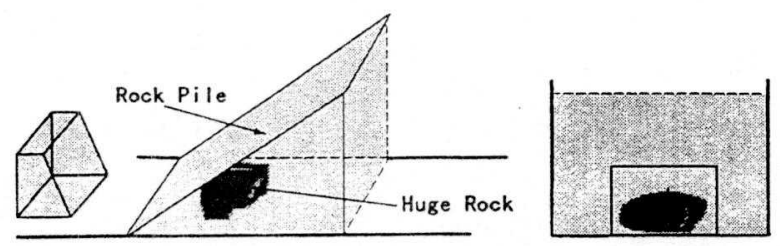

(a) The huge rock is on the bottom of the rock pile.
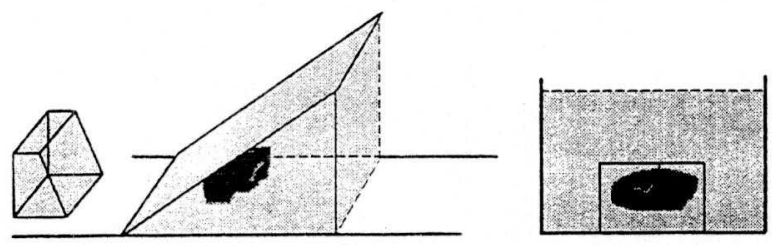

(b) The huge rock is in the middle of the rock pile.
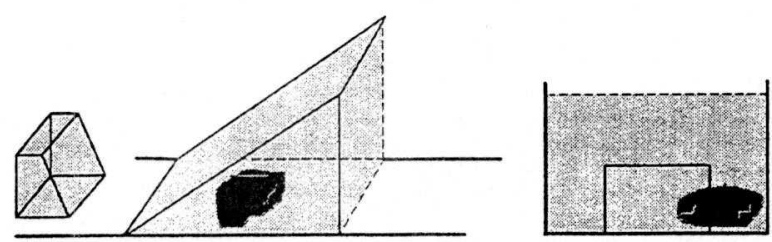

(c) The huge rock is in the side of the rock pile.

\section{Fig.10 Location of huge rock in the rock pile}

\section{CONCLUSIONS}

(1) In order to calculate the resistive forces acting on the bucket, some parameters such as the friction coefficient between the bucket and rocks are necessary. In this study, some equations to predict the friction coefficient between the bucket and rocks, the friction angle among rocks, and the particle diameters were obtained by using the measured resistive forces. These parameters will be used to calculate the resistive forces acting on the bucket in the scooping task.

(2)It became enable to predict the existence of the huge rock in the rock pile by learning the resistive forces which can be measured at the beginning of the scooping task.

\section{Reference}

[1]M.Konaka : Proc. of the 2nd Symposium on Construction Robotics in Japan, pp.1-6, 1991

[2]H.Takahashi et al. : Proc. of the 5th Symposium on Construction Robotics in Japan, pp.19-26, 1995

[3]A.Hemami et al. : Proc. of the 1st Int. Symposium on Mine Mechanization and Automation, pp.1/33-1/44, 1993

[4]H.Takahashi et al. : Transactions of Japan Society of Mechanical Engineers, In Press 


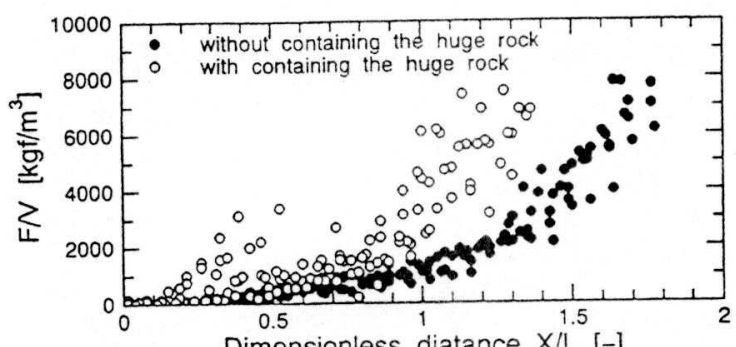

Dimensionless diatance $X / L[-]$

(a)

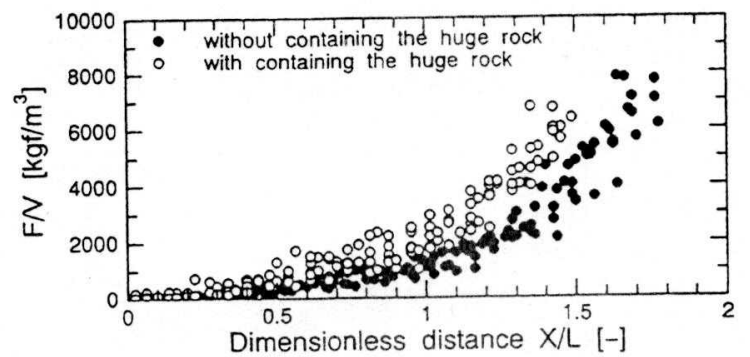

(b)

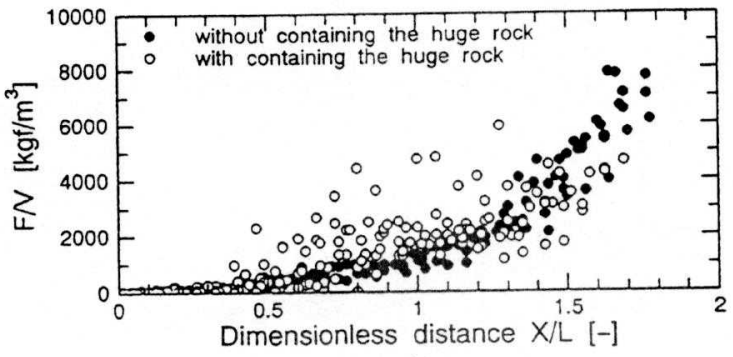

(c)

Fig.11 Relation between $\mathrm{X} / \mathrm{L}$ and $\mathrm{F} / \mathrm{V}$ at several location of huge rock 